\title{
Modeling and Evaluation of the Joint Prevention and Control Mechanism for Curbing COVID-19 in Wuhan
}

\author{
Linhua Zhou, Xinmiao Rong, Meng Fan, Liu Yang, Huidi Chu, Ling Xue, et al. \\ [full author details at the end of the article]
}

Received: 15 June 2021 / Accepted: 30 November 2021 / Published online: 4 January 2022

(C) The Author(s), under exclusive licence to Society for Mathematical Biology 2021

\begin{abstract}
The spread of COVID-19 in Wuhan was successfully curbed under the strategy of "Joint Prevention and Control Mechanism." To understand how this measure stopped the epidemics in Wuhan, we establish a compartmental model with time-varying parameters over different stages. In the early stage of the epidemic, due to resource limitations, the number of daily reported cases may lower than the actual number. We employ a dynamic-based approach to calibrate the accumulated clinically diagnosed data with a sudden jump on February 12 and 13. The model simulation shows reasonably good match with the adjusted data which allows the prediction of the cumulative confirmed cases. Numerical results reveal that the "Joint Prevention and Control Mechanism" played a significant role on the containment of COVID-19. The spread of COVID-19 cannot be inhibited if any of the measures was not effectively implemented. Our analysis also illustrates that the Fangcang Shelter Hospitals are very helpful when the beds in the designated hospitals are insufficient. Comprised with Fangcang Shelter Hospitals, the designated hospitals can contain the transmission of COVID-19 more effectively. Our findings suggest that the combined multiple measures are essential to curb an ongoing epidemic if the prevention and control measures can be fully implemented.
\end{abstract}

Keywords COVID-19 $\cdot$ Modeling with stages $\cdot$ Joint prevention and control mechanism · Data calibration · Dynamical approach · Fangcang Shelter Hospital

Mathematics Subject Classification 92D30 - 34A34

Linhua Zhou and Xinmiao Rong have contributed equally to this work.

Extended author information available on the last page of the article 


\section{Introduction}

The outbreak of the deadly and highly infectious COVID-19 caused by SARS-CoV-2 has been impacting the world since the beginning of 2020 (Chen et al. 2020c) and two waves or even three waves of COVID-19 outbreak (Nørgaard et al. 2021) have hit many countries. The vaccine for SARS-CoV-2 provides hope for a path out of the pandemic, while due to the factors such as limited vaccine resources (Alaran et al. 2021), low willingness of vaccination injection (Sun et al. 2021), and mutations of the virus (Robinson et al. 2021), the control of this epidemic is still facing great difficulties and challenges (Eaton 2021). It is worth noting that the COVID-19 epidemic, which was first reported in Wuhan, was successfully controlled within a few months. Meanwhile, about 50,340 of the 9.04 million people in Wuhan were diagnosed (Office of Health Emergency 2020), this figure is in sharp contrast with the high infection rates in most of the other parts of the world. In fact, evidences show that in response to such a sudden outbreak, in the absence of vaccines, drugs, or even effective diagnostic reagents, the policy of "Joint Prevention and Control Mechanism" (JPCM) proposed by the national authorities has played a significant role in the successful control of Wuhan's COVID-19 epidemic (Chen et al. 2020a; Pang et al. 2020; Huo et al. 2020).

The JPCM is a multi-departmental working mechanism to control the COVID-19 epidemic. In order to mitigate the epidemic, the associated departments developed a series of prevention and control measures, which includes shutdown of public transport service, building of designated hospital (DH) and Fangcang Shelter Hospital (FSH), tracing and home quarantine of close contacts, centralizing all resources from other regions of China, etc. Hubei Daily (2020), Prevention and Control Dynamics, Xinhuanet (2020). With the establishment of more and more DHs and FSHs, and supporting medical staff and equipment throughout the country, the JPCM subsequently developed into instructional principles such as "early detection, reporting, quarantine and treatment," "all persons in need have been tested, quarantined, hospitalized or treated" to ensure all of those different measures could jointly affect the containment of COVID-19 in Wuhan (Yan and Zhao 2020). Indeed, with the gradual implementation of the most thorough joint prevention and control measures, the spread of COVID-19 in Wuhan has been effectively controlled (Xin et al. 2021).

Many available modeling studies of COVID-19 in Wuhan have been contributed to understand the transmission mechanism ( $\mathrm{Li}$ et al. 2020b; Zhao et al. 2020), to predict of the development of the outbreak (Liu et al. 2020a; Tang et al. 2020), and to explore the effectiveness of various nonpharmaceutical intervention (NPIs) strategies (Matteo and Jessica 2020; Ming et al. 2020; Li et al. 2020c, d; Rong et al. 2020). The impact of lockdown in Wuhan on the spread of COVID-19 was widely explored (Kraemer et al. 2020; Pan et al. 2020; Sun et al. 2020), the results showed that the lockdown of Wuhan reduced the propagation of cases to other cities in Hubei province and throughout China. The study in Rong et al. (2020) revealed that the medical resources supply and diagnosis efficiency are essential for an early diagnosis and timely treatment, and improving the timely diagnosis rate can significantly reduce the transmission risk and effectively prevent the endemic of COVID-19. Moreover, the studies on other measures implied that the traffic and community restrictions reduced the flow of population and the spread of disease (Liu et al. 2020b; Fang et al. 2020), 
increasing wards and beds and medical personnel reduced the incidence of severe cases and mortality (Matteo and Jessica 2020; Ming et al. 2020; Li et al. 2020d; Rong et al. 2020), the establishment of the FSHs provided a good isolation and monitoring environment, and further reduced the spread and fatality of the disease ( $\mathrm{Li}$ et al. 2020c; Chen et al. 2020b). Furthermore, assessment for two combined control measures was employed to investigate the effects of the measures on the control of COVID-19. For example, Tang et al. (2020) and Kucharski et al. (2020) found that interventions such as enhanced contact tracing, quarantine, and isolation can effectively reduce the risk of transmission. Leung et al. used a susceptible-infectious-recovered model to describe the potential impact of reducing the intensity of prevention and control measures after the first wave to predict the risk of a second wave of infection (Leung et al. 2020). None of these aforementioned models accounted for the mechanism of the joint containment measures to control COVID-19 in Wuhan. Hence, it is essential to explore how these containment measures worked jointly leading to the control of COVID-19 in Wuhan, or the possible scenario if any of these measures was absent.

In addition, the authorities released eight different versions of prevention and control protocols to make timely changes of the control policies, while the diagnostic criteria for COVID-19 were updated eight times. A shocking scenario of daily reported cases is that the newly confirmed cases surged dramatically on February 12, 2020 (National Health Commission of the People's Republic of China 2020b). Obviously, such a large number of cases cannot be infected within one day, rather they represent an accumulation of clinically diagnosed cases in the previous stage (The State Council Information Office of the People's Republic of China 2020) based on the latest version of diagnostic criteria at that time. Most studies ignore this disparity or use the number of cases after February 12 to fit with their model. No doubt, the issue of cases underreporting may cause bias in predication. Parameters estimation based on precise data can contribute to a realistic assessment of the disease transmission characteristics and a reasonable evaluation of the used control measures (Lin et al. 2020). For example, Lin et al. (2020) filled the missing data before January 15, 2020. Based on the adjusted data, their model successfully captures the course of the COVID-19 outbreak, and sheds light on understanding the trends of the outbreak. Wang et al. (2021) also pointed out that the proposed method partially corrected the under-reporting issue. Therefore, it is meaningful to generate a new method to calibrate the sudden reported data, especially based on the transmission mechanism of COVID-19.

A full understanding of SARS-CoV-2 transmission patterns and risk factors is crucial to plan targeted COVID-19 responses to inform decision making. In this work, based on the transmission of COVID-19 and epidemic characteristics of Wuhan (Prevention and Control Dynamics, Xinhuanet 2020; Chen et al. 2020a; Pan et al. 2020; Hao et al. 2020), we proposed a four-stage transmission model for COVID-19 in Wuhan to reflect the developing control strategies over different stages. Then, we calibrated the under-reported data by a new proposed dynamics-based inversion method. Based on the parameterized model, we simulated the transmission of COVID-19 in Wuhan, and quantify the implementation effects of DH, FSH, home quarantine, and infectious tracing, to examine how the combined measures of JPCM work comprehensively on the controlling of COVID-19. 


\section{Materials}

\subsection{Data}

When lockdown started in Wuhan on January 23, 2020, the JPCM was successively implemented to prevent and control the COVID-19 epidemic. In order to characterize the early transmission dynamics of COVID-19 and to assess the effectiveness of the containment strategy and comprehensive intervention measures, we only concentrate on the data from January 23 to February 25.

The daily reported data of COVID-19 in Wuhan was summarized from the official website of the Wuhan Municipal Health Commission (WMHC) Prevention and Control Dynamics, Xinhuanet (2020). Data information included the daily numbers of newly confirmed cases, cumulative recovered cases, and cumulative infection death (see Fig. 1). From Fig. 1, it is observed that, after the lockdown on Wuhan, the daily new confirmed cases admitted an increasing trend until February 5 and then a decreasing trend.

In addition, the newly confirmed cases surged dramatically on February 12 and 13 with 12523 and 3910 cases, respectively. This "sudden jump" was attributed to a change of test standards in the "Diagnosis and Treatment Protocol for Novel Coronavirus Pneumonia (Trial version 5)" released by National Health Commission. According to the diagnostic criteria for Wuhan in the 5th edition of protocol, from February 12 , all patients had mild clinical symptoms should be reported as confirmed cases together with the RNA test diagnosed cases. The accumulated cases with mild clinical symptoms before February 12 were reported in those days.

The DHs and FSHs were recognized to play a crucial and significant role in the control of COVID-19 (Chen et al. 2020b). There was a severe shortage of hospital beds in the early stage as the number of infections surged. By directing resources to Wuhan, the number and capacity of DHs and FSHs gradually expanded and the number of hospital beds quickly increased. Wuhan had thousands of spare beds in DHs and FSHs till February 25. So the number of new hospital beds in DHs and FSHs can be used to quantitatively assess the efficacy of medical resources. The numbers of hospital beds in DHs and FSHs were collected and estimated from the data of hospital diagnoses included in public reports from the Wuhan COVID-19 Epidemic Prevention and Control Headquarters (Prevention and Control Dynamics, Xinhuanet 2020) (see Fig. 2).

The COVID-19 outbreak in Wuhan was across several different stages defined by significant events and interventions. The measures and strength of prevention and control, in particular the building and addition of new hospital beds, highly varied through multiple stages. The values of key parameters also varied during different stages. Considering the time-varying strength of control measures, we will estimate the parameters in different stages, independently and separately. Our modeling study, which incorporates the estimated time-varying parameters, will delineate the fullspectrum transmission dynamics of the COVID-19 outbreak in Wuhan, and evaluate the effect of the JPCM in different stages. 


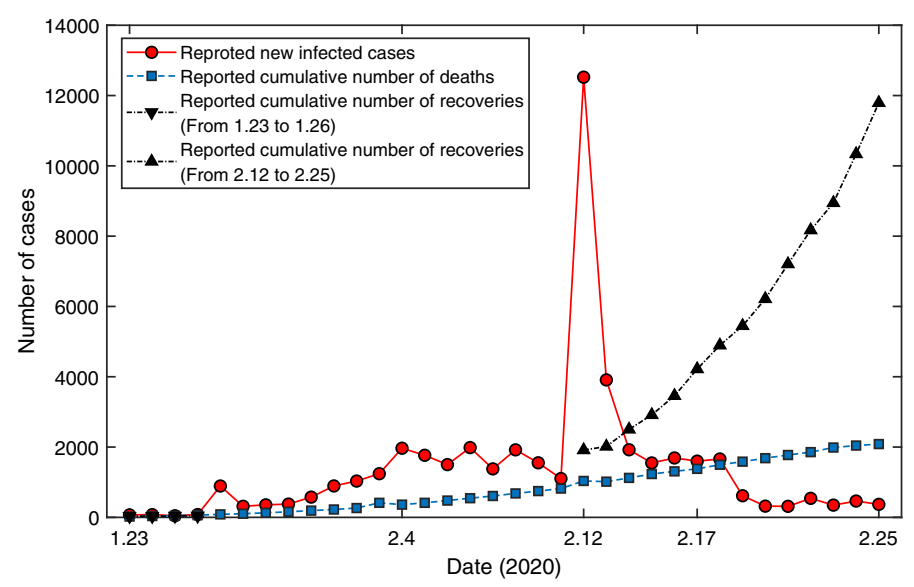

Fig. 1 Daily reported data of new confirmed cases, cumulative death, and cumulative recovery of COVID19 in Wuhan from January 23 to February 25. The daily reported data of new confirmed cases prior to the "sudden jump" on February 12 is to be calibrated. Data source: Prevention and Control Dynamics, Xinhuanet (2020)

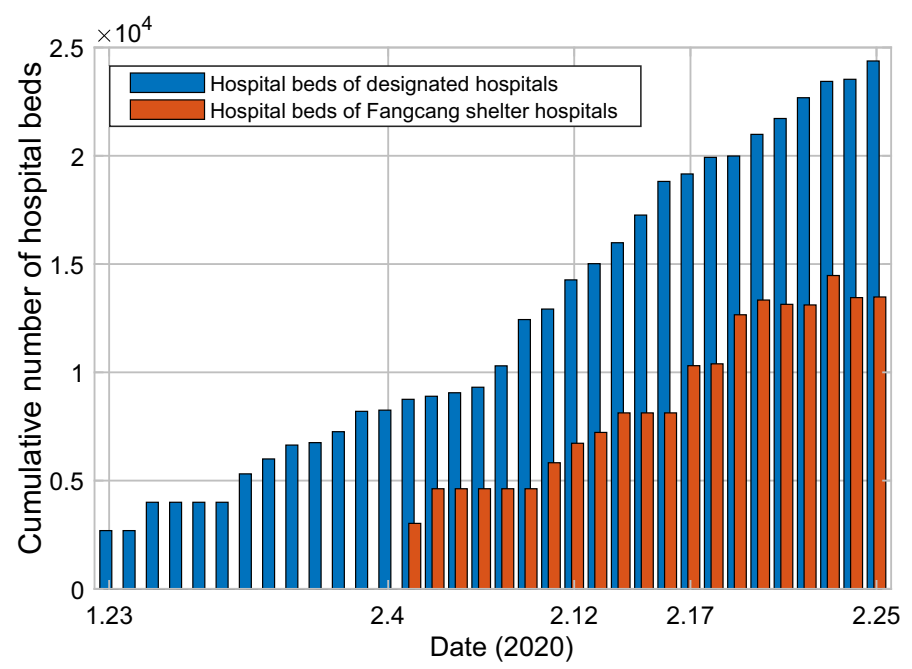

Fig. 2 Cumulative number of hospital beds in DHs and FSHs from January 23 to February 25 in Wuhan

\subsection{Stages of COVID-19 Outbreak in Wuhan Considering JPCM}

During the outbreak of COVID-19, the resources to mitigate the epidemic vary greatly in different periods. The prevention and control measures have been implemented rapidly, from the early stages in Wuhan and other key areas of Hubei, to the overall national epidemic. From January 23 to February 25, 2020, Wuhan's fight against the epidemic can be divided into four different stages, with several important events defining those stages following the guidelines of JPCM: 
Stage I Initial Progress in Containing the Virus (January 23 to February 4, 2020). January 23 is the date when Wuhan was put on a lockdown (Prevention and Control Dynamics, Xinhuanet 2020) and Huoshenshan Hospital started to be built (Routine press conference on the prevention and control of the COVID-19 epidemic 2020). It is an initial phase of the outbreak due to the growing emergence of large numbers of infections after the lockdown. It is crucial to note that the lack of medical resources is one of the key factors leading to the increase in infections. In this stage, the family-based household quarantine is the main measures to control the COVID-19.

Stage II FSHs and DHs were available to admit infections (February 5 to February 12, 2020).

Huoshenshan Hospital was completed to admit confirmed infected patients with severe symptoms on February 4 (Health Commission of Hubei Province 2020b), and the first bed in FSH is opened to treat quarantine individuals with mild symptoms on February 5 (Routine press conference on the prevention and control of the COVID-19 epidemic 2020). Leishenshan Hospital was officially launched on February 8 (Health Commission of Hubei Province 2020c). A number of hospital beds in DH and FSH were available and the stalemate situation in curbing the epidemic is essentially changed. As of 12 February, the number of hospital beds of DHs and FSHs is about 14269 and 8605, respectively (see Fig. 2).

Stage III Early detection, reporting, quarantine, and treatment (February 13 to February 17, 2020).

On February 13, all the residential communities in Wuhan were under lockdown and 24-h closed management was implemented in all residential areas (Health Commission of Hubei Province 2020d). More DHs and FSHs were built consequently, the sufficient hospital beds could be applied for the confirmed mild infections and severe patients through contacting tracing and testing. The FSHs provided timely treatment for the mild cases, as well as breaking down transmission of the virus.

Stage IV Overall Every Control and Clear approach (February 18 to February 25, 2020).

On February 18, the most thorough, rigorous, and comprehensive prevention and control measures were officially and legally adopted to ensure that all those in need have been tested, quarantined, hospitalized, or treated as much as possible. The Overall Every Control and Clear approach (Hao et al. 2020) was applied in nucleic acid testing and the testing efficiency was greatly enhanced. Great effort had been exhaustively applied in epidemiological investigations to find every infected person and track every close contact for quarantine. The implementation of such strategy significantly controlled the source of infection and quickly suppressed the epidemic. 


\section{Modeling}

\subsection{Time-Varying Model Formulation}

During the COVID-19 outbreak, most people in Wuhan were observing home quarantine which puts family members at risk, and $75-80 \%$ of all clustered infections were within families, suggesting high rates of family transmission (Chen et al. 2020b). We classify the population into the following ten compartments: susceptible $(S(t))$, close contact $(C(t))$, uninfected under home quarantine $(U(t))$, asymptomatic infectious under home quarantine $(A(t))$, infectious with mild symptoms under home quarantine $(Q(t))$, infectious with severe symptoms under home quarantine $(I(t))$, infectious in $\mathrm{DH}(H(t))$, infectious in FSH $(M(t))$, recovered $(R(t))$, and deaths due to COVID-19 $(D(t))$. The epidemic is assumed to take place on a timescale of days.

The susceptible individuals could be infected via contact with the infectious $A, Q, I$ and then move to the compartment $C$ at rate of $\left(\sigma^{A} A+\sigma^{Q} Q+\sigma^{I} I\right) S / N$ by contact tracing. After the observation period (i.e., incubation period) $1 / v$ days, the close contact $C$ become infectious and move to the infectious $A, Q, I$ at rate $\beta^{*} \nu C$ with probabilities $(1-\rho), \rho(1-\xi), \rho \xi$, respectively, also move to the uninfected self-quarantined $U$ at rate $\left(1-\beta^{*}\right) \nu C$. The uninfected self-quarantined $U$ becomes susceptible after quarantined period $1 / \eta$ days. The infectious $A, H$, and $M$ can recover at rate $\gamma^{A}, \gamma^{H}$ and $\gamma^{M}$, respectively. The COVID-19 deaths only occur in DH at rate $\mu H$.

A schematic description of the transmission dynamics of COVID-19 is depicted in Fig. 3. The flow diagram in Fig. 3 leads to the following model

$$
\left\{\begin{array}{l}
\frac{\mathrm{d} S}{\mathrm{~d} t}=-\frac{\left(\sigma^{A} A+\sigma^{Q} Q+\sigma^{I} I\right) S}{S+C+U+A+Q+I+H+R+D+M}+\eta U, \\
\frac{\mathrm{d} C}{\mathrm{~d} t}=\frac{\left(\sigma^{A} A+\sigma^{Q} Q+\sigma^{I} I\right) S}{S+C+U+A+Q+I+H+R+D+M}-v C, \\
\frac{\mathrm{d} U}{\mathrm{~d} t}=\left(1-\beta^{*}\right) \nu C-\eta U \\
\frac{\mathrm{d} A}{\mathrm{~d} t}=\beta^{*}(1-\rho) v C-\gamma^{A} A-f_{4}(t, A), \\
\frac{\mathrm{d} Q}{\mathrm{~d} t}=\beta^{*} \rho(1-\xi) \nu C-f_{2}(t, Q)-f_{3}(t, Q), \\
\frac{\mathrm{d} t}{\mathrm{~d} H}=\beta^{*} \rho \xi \nu C-f_{1}(t, I), \\
\frac{\mathrm{d} t}{\mathrm{~d} t}=f_{1}(t, I)+f_{2}(t, Q)-\gamma^{H} H-\mu H, \\
\frac{\mathrm{d} D}{\mathrm{~d} t}=\mu H \\
\frac{\mathrm{d} M}{\mathrm{~d} t}=f_{3}(t, Q)+\gamma^{H} H+\gamma^{M} M,
\end{array}\right.
$$

where the variables/parameters and their epidemiological significance, initial/default values, and reference sources are summarized in Tables 1 and 2. 


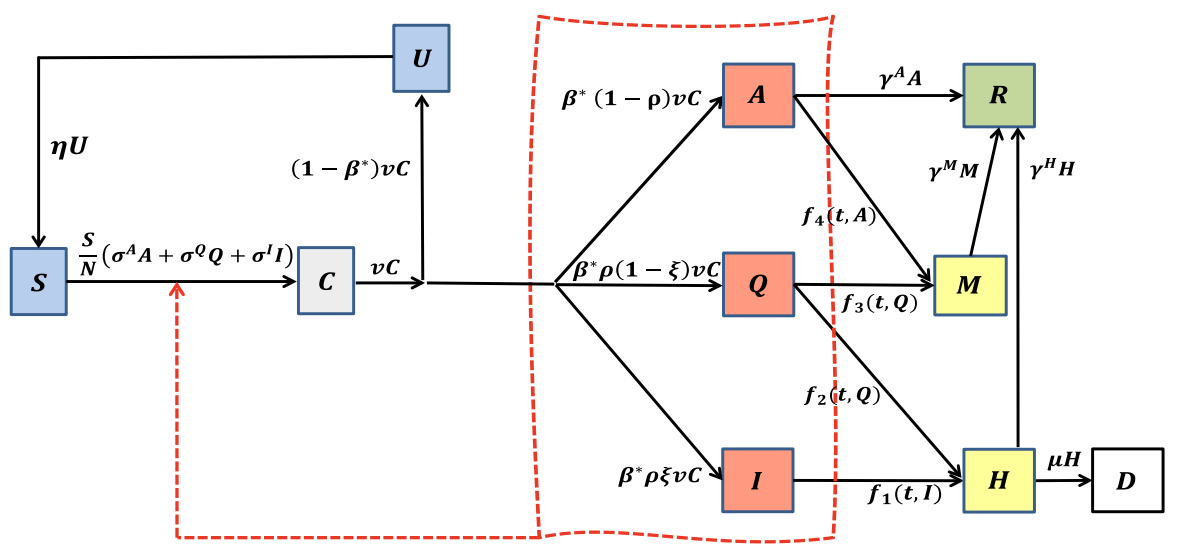

Fig. 3 Flow diagram of the transmission of COVID-19

Table 1 Variables and their descriptions, initial values, and sources

\begin{tabular}{|c|c|c|c|}
\hline Variable & Descriptions & Initial values & Source \\
\hline$S(t)$ & Number of susceptible & $9 \times 10^{6}$ & People's Network (2020) \\
\hline$C(t)$ & Number of close contact & 1302 & Estimated \\
\hline$U(t)$ & $\begin{array}{l}\text { Number of uninfected under home } \\
\text { quarantine }\end{array}$ & 0 & $\begin{array}{l}\text { Calculated Yang et al. (2020), Health } \\
\text { Commission of Hubei Province } \\
(2020 \mathrm{a})\end{array}$ \\
\hline$A(t)$ & $\begin{array}{l}\text { Number of asymptomatic infec- } \\
\text { tious under home quarantine (who } \\
\text { will never develop symptoms) }\end{array}$ & 16 & $\begin{array}{l}\text { Yang et al. (2020), Health Commission } \\
\text { of Hubei Province (2020a) }\end{array}$ \\
\hline$Q(t)$ & $\begin{array}{l}\text { Number of infectious with mild } \\
\text { symptoms under home quarantine }\end{array}$ & 245 & $\begin{array}{l}\text { Yang et al. (2020), Health Commission } \\
\text { of Hubei Province (2020a) }\end{array}$ \\
\hline$I(t)$ & $\begin{array}{l}\text { Number of infectious with severe } \\
\text { symptoms under home quarantine }\end{array}$ & 89 & $\begin{array}{l}\text { Yang et al. (2020), Health Commission } \\
\text { of Hubei Province (2020a) }\end{array}$ \\
\hline$H(t)$ & $\begin{array}{l}\text { Number of infectious in Desig- } \\
\text { nated Hospitals }\end{array}$ & 30 & $\begin{array}{l}\text { Calculated Yang et al. (2020), Health } \\
\text { Commission of Hubei Province } \\
(2020 \mathrm{a})\end{array}$ \\
\hline$M(t)$ & $\begin{array}{l}\text { Number of infectious in Fangcang } \\
\text { Shelter Hospitals }\end{array}$ & 0 & Assumed \\
\hline$R(t)$ & Number of recovered & 28 & $\begin{array}{l}\text { Calculated Health Commission of } \\
\text { Hubei Province (2020a) }\end{array}$ \\
\hline$D(t)$ & $\begin{array}{l}\text { Number of death due to COVID- } \\
19\end{array}$ & 17 & $\begin{array}{l}\text { Health Commission of Hubei Province } \\
\text { (2020a) }\end{array}$ \\
\hline
\end{tabular}

In (3.1), $f_{i}, i=1,2,3,4$, represent the transmission functions between different compartments. Next, we deliberately discuss those functions in detail. Note that, the hospital beds in $\mathrm{DH}$ are preferred for treating patients with severe symptoms, and then sparse beds can be used for infections with mild symptoms; the hospital beds in FSHs are mainly served to isolate and care for patients with mild to moderate symptoms, and sparse beds are available for asymptomatic infections. Let $B_{D H}(t)$ and $B_{M H}(t)$ 


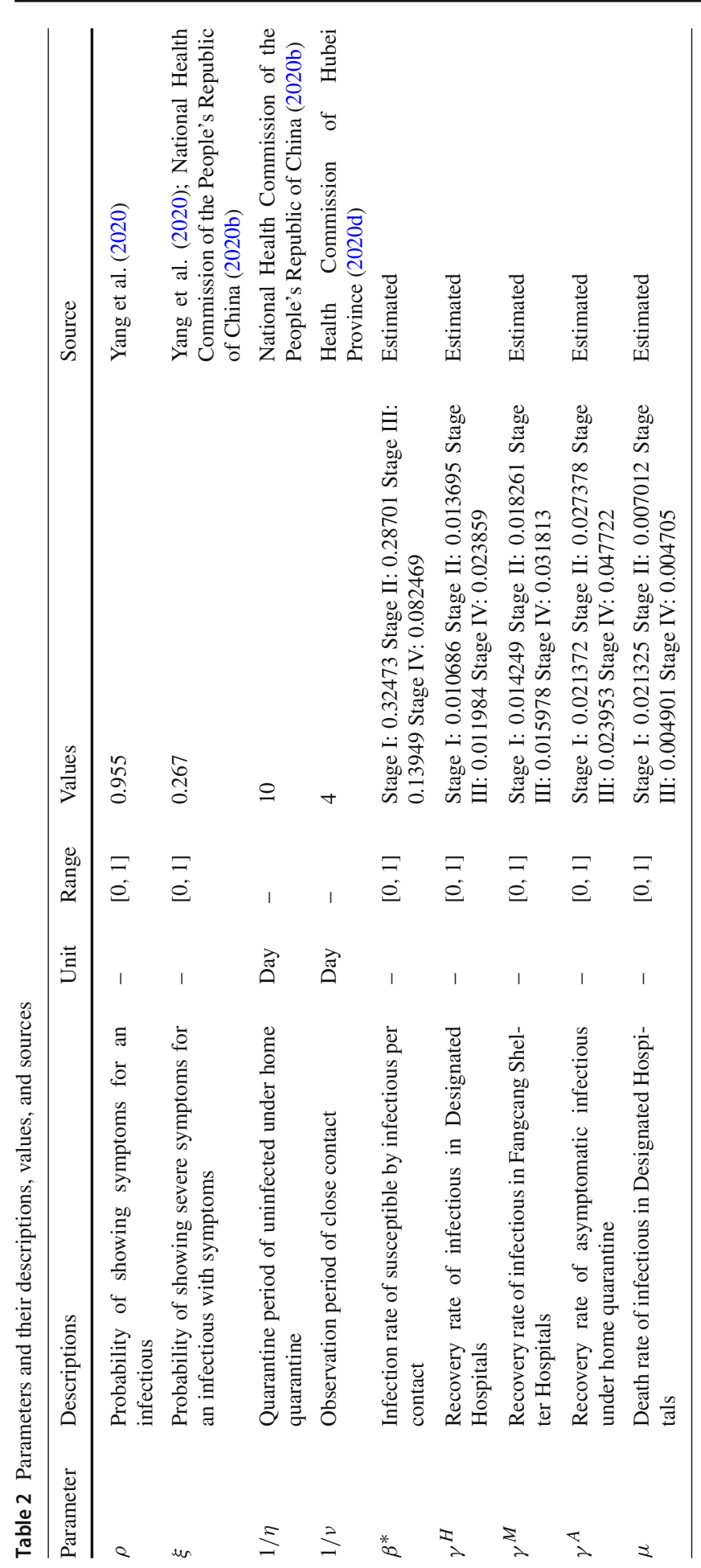




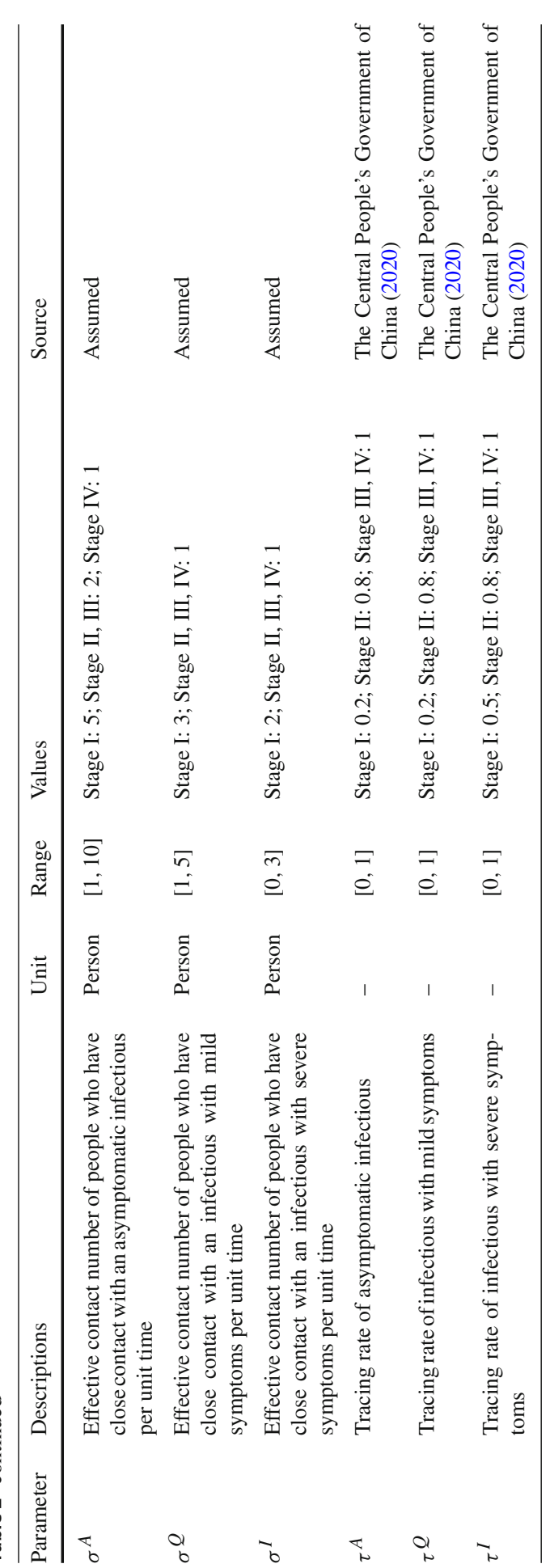


denote the numbers of hospital beds in $\mathrm{DH}$ and $\mathrm{FSH}$ at time $t$, respectively. Then, we have the following facts:

- The number of infectious individuals with severe symptoms under home quarantine being admitted into $\mathrm{DH}$ per unit time

$$
f_{1}(t, I)=\min \left\{\tau^{I} I(t), \max \left\{B_{D H}(t)-H(t), 0\right\}\right\},
$$

- The number of infectious individuals with mild symptoms under home quarantine being admitted into $\mathrm{DH}$ per unit time

$$
f_{2}(t, Q)=\min \left\{\tau^{Q} Q-f_{3}(t, Q), \max \left\{B_{D H}(t)-H(t), 0\right\}-f_{1}(t, I)\right\},
$$

- The number of infectious individuals with mild symptoms under home quarantine being admitted into FSH per unit time

$$
f_{3}(t, Q)=\min \left\{\tau^{Q} Q, \max \left\{B_{M H}(t)-M(t), 0\right\}\right\},
$$

- The number of asymptomatic infectious under home quarantine being admitted into FSH per unit time

$$
f_{4}(t, A)=\min \left\{\tau^{A} A, \max \left\{B_{M H}(t)-M(t), 0\right\}-f_{3}(t, Q)\right\},
$$

where $\tau^{I}, \tau^{Q}$, and $\tau^{A}$ are the tracing rate of compartments $I, Q$, and $A$, respectively.

\subsection{Under-Reported Data Calibration}

Under-reported data are commonly found as a crucial issue for data collection, especially for a new emerging infectious disease that has never appeared before. At the early stages of COVID-19 outbreak after Wuhan's lockdown, the reported number of confirmed cases is not the actual number of infections on a specific day due to the limited capability of nucleic acid test. In addition, the diagnostic criteria for COVID19 were updated continually. Before February 12, the National Health Commission (NHC) of China employed nucleic acid test as the unique diagnostic standard to confirm infected cases. On February 12, the clinically diagnosed cases together with the nucleic acid test diagnosed cases were first reported as confirmed cases according to the latest version of prevention and control protocols at that time. As a result, the daily number of infected cases in Wuhan has jumped with significantly large sizes on February 12 and 13 with 12,523 and 3910 cases, respectively (see Fig. 1).

In the previous modeling study of COVID-19, only the data from February 12 onward were used to estimate the model parameters ( $\mathrm{Li}$ et al. 2020c), or the data of February 12 and 13 were deliberately neglected (Li et al. 2020a), or adjusted data through the statistic calibration procedure which fits an exponential function (Wang et al. 2021). The analysis of under-reported data may lead to a biased estimation and 
prediction when mathematical or statistical models are used. How to deal with and correct the under-reporting data, in particular the significantly sudden jumps, is not only crucially important but also very challenging.

Obviously, the new reported clinically diagnosed cases on February 12 and 13 cannot happen within one day, rather than they represent an accumulation of cases that have not been reported and timely diagnosed by RNA test before February 12 . Therefore, the daily increment of clinically diagnosed cases should also follow the same rule of the reported increment confirmed by the RNA test. The method developed in Wang et al. (2021), Li et al. (2020a) for adjusting the under-reported data assumes that the data obeys certain distributions (exponential or gamma distribution), while this assumption may enlarge the data biases. In reality, the fitted evolution pattern of those cases with heavy symptoms (i.e., RNA-Test confirmed cases) follows the laws of the transmission mechanism of COVID-19 in Wuhan. Then, based on model (3.1), we develop a dynamics-based data inversion approach to calibrate the number of infections. The approach proceeds through the following steps.

Step 1 With the help of the COVID-19 transmission model (3.1), by employing the nonlinear least squares procedure, we formulate the evolution pattern of the data of daily new confirmed cases diagnosed by RNA test from January 23 to February 13 (see the fitted curve in Fig. 4a). The number of fitted daily new confirmed cases (see the model output in Fig. 4a) are denoted by $x_{1}, x_{2}, \ldots, x_{22}$, respectively.

Step 2 Following the fitted evolution pattern obtained in Step 1, the sudden increments of the clinically diagnosed cases on February 12 and 13, which are denoted by $\mathbb{M}_{1}$ and $\mathbb{M}_{2}$, respectively, are inversely distributed into each day from January 23 to February 13, in proportion to the percentage of daily fitting data in all fitting data, that is,

$$
\frac{x_{i}}{\sum_{k=1}^{22} x_{k}}\left(\mathbb{M}_{1}+\mathbb{M}_{2}\right), \quad i=1,2, \ldots, 22
$$

The calibrated data are shown in Table 3 and are illustrated by the solid black curve with "o" in Fig. 4b. For example, on February 1, the reported number of daily new infected cases is 894 , while the calibrated number is 1479 , with a difference of 585 cases. It means that, on February 1894 cases were confirmed by RNA test, while 585 cases were not since those individuals just had mild clinical symptoms and were not included in the reported number according to the prevention and control protocols at that time.

\subsection{Parameters Estimation}

We set the initial values of state variables and the values of parameters for each stage in the model (3.1). Some of those values are extracted from the relevant literature and have been widely recognized, some are assumed based on the publicly published data of infections in Wuhan or well-accepted epidemiological facts, and the others are estimated by fitting the model outcomes to the data using least square method. 
Table 3 Calibrated data of daily new infected cases

\begin{tabular}{llllll}
\hline Date & January 23 & January 24 & January 25 & January 26 & January 27 \\
Reported Cases & 70 & 77 & 46 & 80 & 892 \\
Calibrated Cases & 151 & 182 & 177 & 243 & 1094 \\
Date & January 28 & January 29 & January 30 & January 31 & February 1 \\
Reported Cases & 315 & 356 & 378 & 576 & 894 \\
Calibrated Cases & 565 & 665 & 760 & 1049 & 1479 \\
Date & February 2 & February 3 & February 4 & February 5 & February 6 \\
Reported Cases & 1033 & 1242 & 1967 & 1766 & 1501 \\
Calibrated Cases & 1757 & 2137 & 3050 & 2841 & 2552 \\
Date & February 7 & February 8 & February 9 & February 10 & February 11 \\
Reported Cases & 1985 & 1379 & 1921 & 1552 & 1104 \\
Calibrated Cases & 3006 & 2369 & 2880 & 2481 & 2003 \\
Date & February 12 & February 13 & & & \\
Reported Cases & 1201 & 1201 & & & \\
Calibrated Cases & 2071 & 2043 & & & \\
\hline
\end{tabular}

(a)

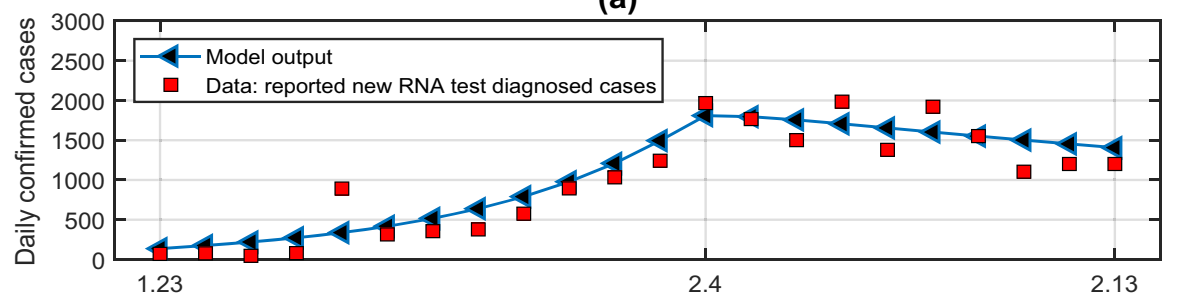

(b)

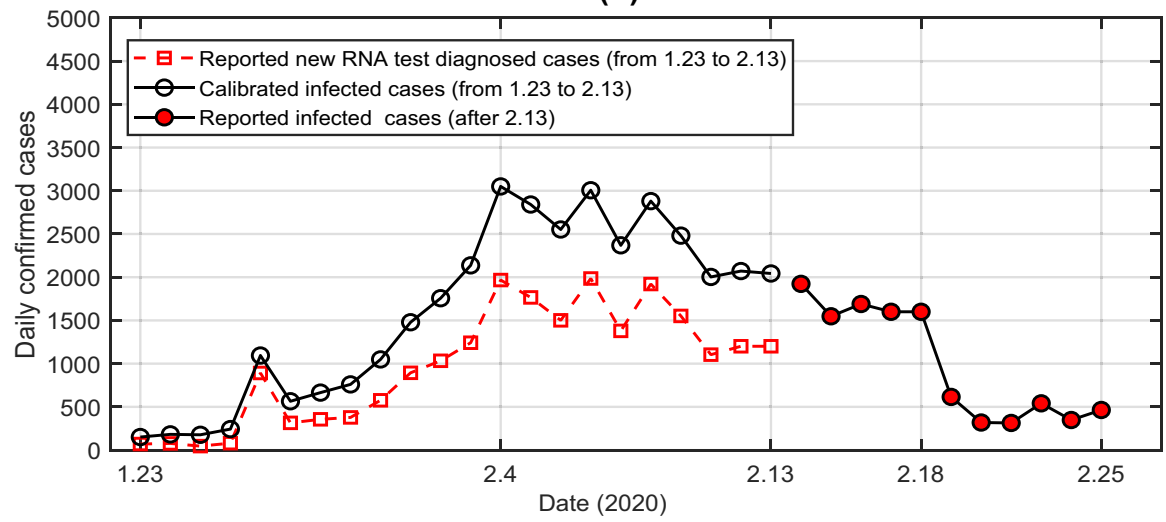

Fig. 4 Calibration of under-reported data of daily new infected cases in Wuhan from January 23 to February 13. a Reported number of daily new confirmed cases by RNA test and the best fitted curve. b Calibrated data of daily new infected cases shown by the solid black curve with "o" 
By the works of Yang et al. (2020), 69.9\%, 25.5\%, and 4.5\% of the infectious would be mild, severe, and asymptotic cases, respectively. Thus, the parameters $\rho=0.955$ and $\xi=0.267$ for (3.1). Since the incubation period of COVID-19 is $1-14$ days, mostly 3-7 days (National Health Commission of the People's Republic of China 2020b), we assume that the observation period of close contact is 4 days (i.e., $v^{-1}=4$ ), and the uninfected under home quarantine (i.e., $U(t))$ remain in isolation for another 10 days then become susceptible again (i.e., $\eta^{-1}=10$ ). The mean duration time between onset of symptoms and diagnosis is 4.95 days at the early stage of COVID19 outbreak (The Central People's Government of China 2020), then we set $\tau^{I}=0.5$ and $\tau^{Q}=0.2$ for the first stage.

Note that the infectious $A(t), Q(t)$, and $I(t)$ are under home quarantine and their main contacts are among family members, considering the typical family structure in Wuhan, the values of intrafamily contacts of $A, Q$, and $I$ are assumed to be $\sigma^{A}=5$, $\sigma^{Q}=2$, and $\sigma^{I}=1 \mathrm{in}$ Stage I, respectively. For Stage II-IV, owing to the successively stricter control measures and the increasing medical supplies, the values of those three parameters are properly adjusted (see Table 2). In Table 1, the initial values of state variables are directly obtained or calculated from public reports and published papers (Yang et al. 2020; People's Network 2020; Health Commission of Hubei Province 2020a).

Consider the function $B_{D H}(t)$ and $B_{M H}(t)$. Let $i=1,2, \ldots, 33$ denote the days from January 23 to February 26. Define

$$
\begin{aligned}
& B_{D H}(t)=d_{i}, \quad t \in[i, i+1), \quad i=1,2, \ldots, 32 \text {, }
\end{aligned}
$$

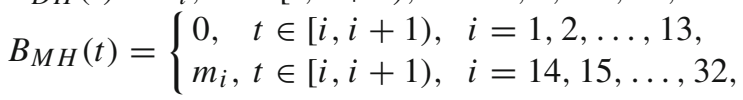

where everyday begins at 0:00 am, $d_{i}$ and $m_{i}$ are the actual number of the hospital beds in DH and FSH of the $i$-th day, respectively (see Fig. 2).

We employ the nonlinear least squares procedure to estimate the initial value of $C(t)$, the infection rate $\left(\beta^{*}\right)$, recovery rate $\left(\gamma^{M}, \gamma^{H}\right.$, and $\left.\gamma^{A}\right)$, and death rate $(\mu)$ in consecutive four stages, respectively. The nonlinear least square estimation is to find the parameter values to minimize the following objective function

$$
\begin{aligned}
& \mathbb{F}\left(\beta^{*}, \gamma^{M}, \gamma^{H}, \gamma^{A}, \mu, C(0)\right)=\sum_{i=1}^{32}\left[\left(C I(i)-\widehat{C I}_{i}\right)^{2}+\left(D(i)-\widehat{C D}_{i}\right)^{2}\right] \\
& \quad+\left[\sum_{i=1}^{4}\left(R(i)-\widehat{C R}_{i}\right)^{2}+\sum_{i=14}^{32}\left(R(i)-\widehat{C R}_{i}\right)^{2}\right]
\end{aligned}
$$

where $\mathbb{F}\left(\beta^{*}, \gamma^{M}, \gamma^{H}, \gamma^{A}, \mu, C(0)\right)$ represents the error between the output of model and the data; $\widehat{C I}_{i}, \widehat{C D}_{i}$ and $\widehat{C R}_{i}$ are the total numbers of the reported cumulative confirmed cases, the death, and recovered cases on the $i-t$ day, respectively; while

$$
\begin{aligned}
& C I(i)=\left.C I(t)\right|_{t=i} \triangleq[I(t)+Q(t)+A(t)+H(t)+M(t)+R(t)+D(t)]_{t=i}, \\
& D(i)=\left.D(t)\right|_{t=i}, R(i)=\left.R(t)\right|_{t=i},
\end{aligned}
$$

$i=1,2, \ldots, 32$, are the corresponding values calculated from the numerical outputs of (3.1). One thing to note is that, with the calibrated data and the high detection 
efficiency in stage III and IV, the sum of the numbers of the infectious under home quarantine $(I(t), Q(t), A(t))$, the treated in hospitals $(H(t), M(t))$, the recovered $(R(t))$ and the death $(D(t))$ is just the total number of the cumulative confirmed cases at time $t$.

All the initial values of state variables and the values of parameters are summarized in Tables 1 and 2, respectively.

\section{Results}

In what follows, by performing some numerical simulations, we simulate our model (3.1) to calibrate the actual data, and validate the parameterized model to assess the effect of joint prevention and control measures of COVID-19.

\subsection{Fitting Results and Predictions}

From the best fitted curves in Fig. 5, it is observed that the model calculation shows reasonably good agreement with the reported data. The validation of the estimation (see the blue solid curve and the green dots in Fig. 5a) also shows that the model parameterized with the calibrated data makes a good prediction. Figure 6 shows the comparisons of the model output $H(t)$ and $M(t)$ with the actual number of available hospital beds in DH and FSH. It also reflects the occupancy of the substantially increasing hospital beds. From Fig. 6a, one observes that the number of available hospital beds in DH is larger than that of the confirmed cases in Stage I owing to various possible reasons such as low efficiency of RNA test, shortage of medical resources, and lack of healthcare personnel; with the increasing of new infection and testing efficiency, there were almost no spare beds in both DH and FSH after February 5; in later Stage IV, there are again sparse beds in DH, but all hospital beds in FSH are used to treat the infected individuals with mild symptoms. Those facts are well consistent with the actual situation (National Health Commission of the People's Republic of China 2020b). The fitting results and predictions are very interesting and exciting and also demonstrate the validity of our parameterized model (3.1).

\subsection{Effect of Hospital Beds in DH and FSH}

The available beds in "Huoshenshan" and "Leishenshan" hospitals for infections with severe symptoms make great sense in the prevention of COVID-19 transmission. If "Huoshenshan" and "Leishenshan" designated hospitals were not built in Wuhan (i.e., the number of available designated hospital beds were reduced by 1000 and 1500 on February 3 and February 8, respectively), then, from Fig. 7, one can find that, by the middle of March, the two designated hospitals, which only contains 2500 beds, have already averted more than 30,000 cases (see the dash curve in Fig. 7).

It is FSH that ensures sufficient capacity to both effectively isolate and care for the large numbers of people who experience mild to moderate COVID-19. If the FSH was not built in Wuhan (i.e., $B_{M H}(t)=0$ ), then, in Fig. 7, the dash-dot curve 


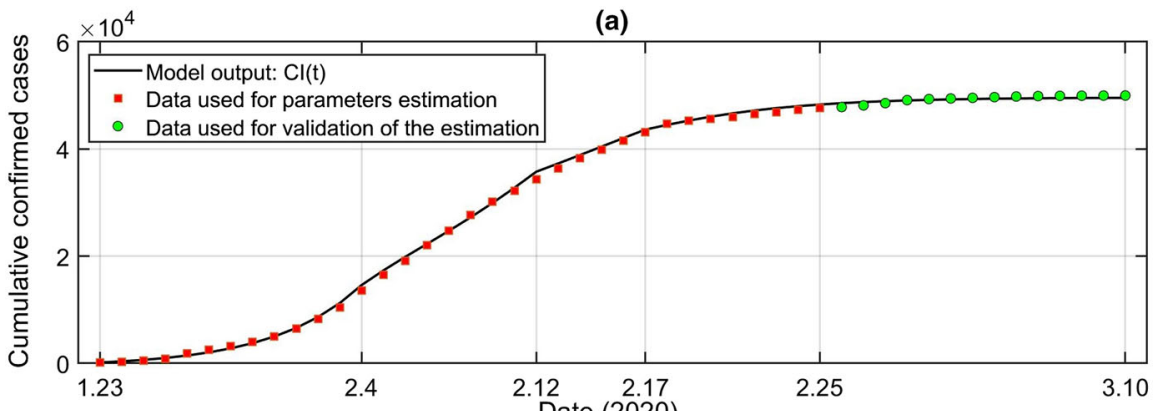

Date (2020)
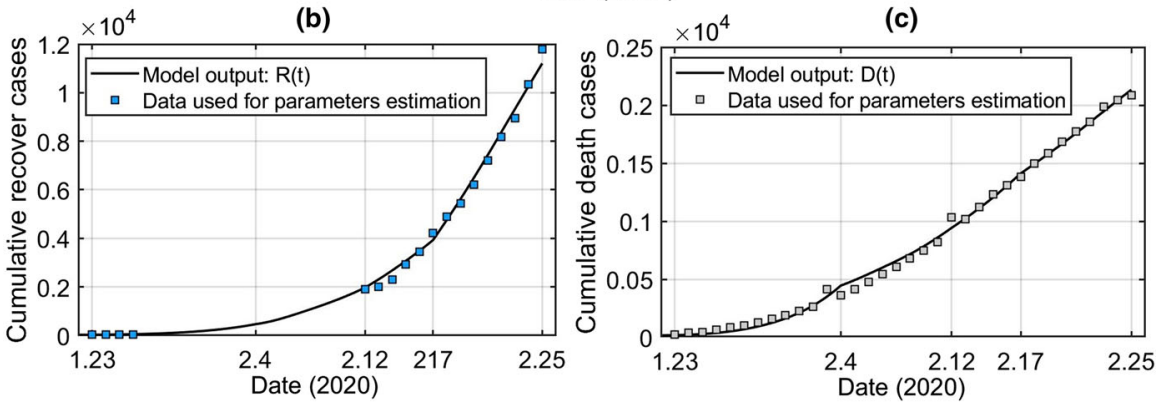

Fig. 5 Comparisons of model outputs, $R(t)$, and $D(t)$ with the daily data. The best fitted curves indicate that our predictions well match the calibrated data of cumulative confirmed cases (a and Fig. 4), and the reported data of cumulative death and cumulative recovery (b-c and Fig. 1) from January 23 to February 25. The validation of the estimation (see the blue solid curve and the green dots in a) from February 26 to March 10 also shows that the parameterized model makes a perfect predication. Here, the data of green dots is the cumulative confirmed cases reported by WMHC (Prevention and Control Dynamics, Xinhuanet 2020) (Color figure online)
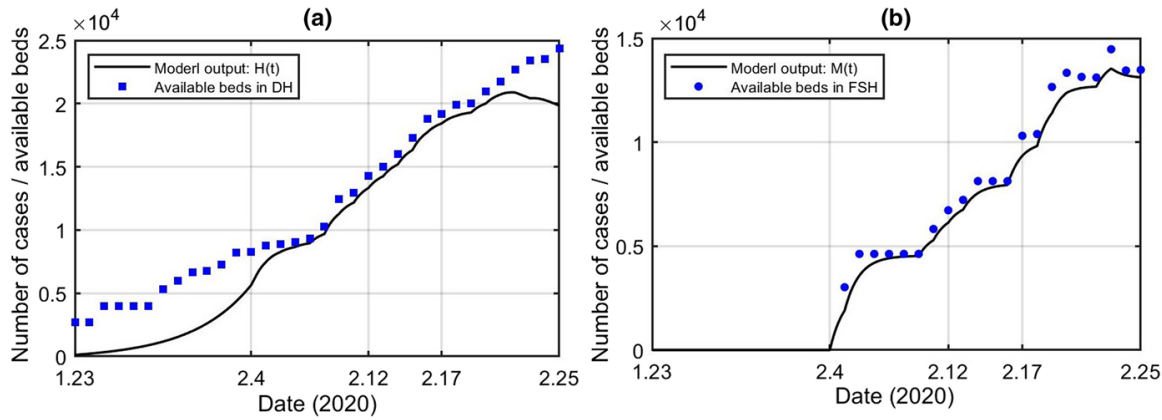

Fig. 6 Comparisons of model outputs $H(t)$ and $M(t)$ with the actual number of available hospital beds in designated hospitals and Fangcang Shelter Hospitals

revealed that the outbreak of COVID-19 in Wuhan would be out of control and the number of cumulative confirmed cases would reach to 140,000 by March 10, even if the prevention and control measures were strengthened.

The sensitivity analysis in Fig. 8 clearly illustrates that the hospital bed capacity has a critical impact on the control of COVID-19 in Wuhan. If the local government 


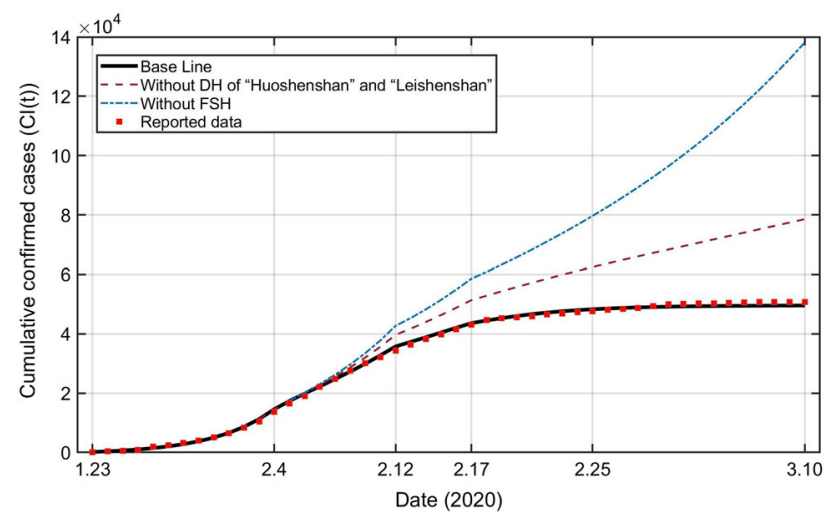

Fig. 7 Comparison of cumulative confirmed cases under different hypothetical control scenarios. It reveals that the DH of "Huoshenshan" and "Leishenshan," and the FSH, have a great impact on the containment of COVID-19 in Wuhan

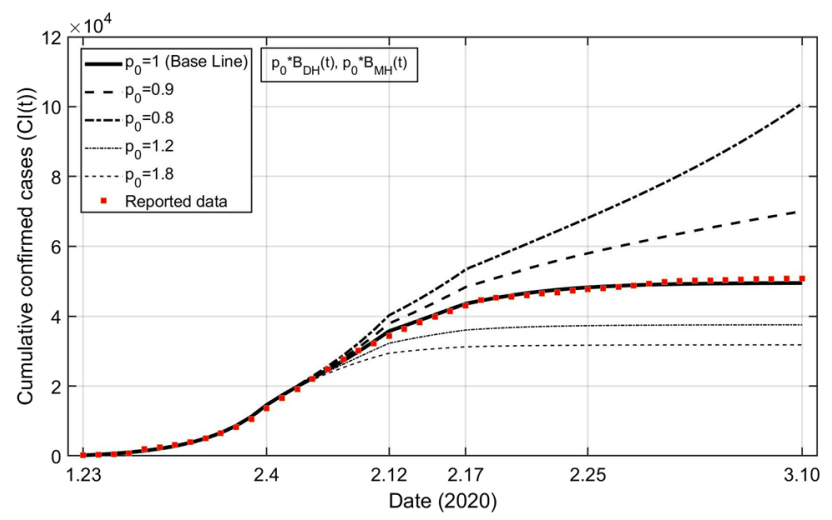

Fig. 8 Sensitivity analysis of hospital bed capacity on COVID-19 in Wuhan. The numbers of available beds in both $\mathrm{DH}$ and FSH are changed by varying parameter $p_{0}$, while other parameters are the same as those in Fig. 5

of Wuhan can't provide the actual available hospital beds (see in Fig. 2) in a short term, for example, the number of hospital beds was decreased by 20 percent (i.e., $0.8 * B_{D H}(t)$ and $\left.0.8 * B_{M H}(t)\right)$, then the cumulative confirmed cases would be far more than the current infection size (see the thick dash-dot curve with $p_{0}=0.8$ and the baseline of red solid curve with $p_{0}=1$ in Fig. 8). On the other hand, if there were more public health resources in February and the number of hospital beds was increased by 20 percent (i.e., $1.2 * B_{D H}(t)$ and $1.2 * B_{M H}(t)$ ), then the final infection size of COVID-19 in Wuhan would be less than 38,000 cases (see the thin dash-dot curve with $p_{0}=1.2$ in Fig. 8). 


\subsection{Effect of Home Quarantine}

After city's lockdown, Wuhan implemented social distancing to inhibit the transmission of COVID-19. Home quarantine of close contact is one of the most important methods of social distancing. The parameters $\sigma^{I}, \sigma^{Q}$, and $\sigma^{A}$ are the numbers of close contact of infectious under home quarantine, with severe symptoms, mild symptoms, and without symptoms per unit time, respectively. The values of $\sigma^{I}, \sigma^{Q}$, and $\sigma^{A}$ are determined by family members, self-protect awareness, medical resources, etc. With the actual capacity of hospital beds in DH and FSH, Fig. 9 shows the sensitivity analysis of home quarantine on the containment of COVID-19 in Wuhan in terms of $\sigma^{I}, \sigma^{Q}$, and $\sigma^{A}$. One finds in Fig. 9 that, the smaller of the numbers of close contact (i.e., $\sigma^{I}, \sigma^{Q}$, and $\sigma^{A}$ ), the smaller of the numbers of cumulative confirmed cases, and there are more spare beds in both $\mathrm{DH}$ and $\mathrm{FSH}$. For example, if the numbers of close contact were decreased by $5 \%$, then the number of cumulative confirmed cases would be reduced to 32,507 (see the thin dash curve with $p_{1}=0.95$ in Fig. 9a). There are again spare beds in DH from February 14 (see the thin dash curve with $p_{1}=0.95$ in Fig. 9b), nine days ahead of the actual circumstance (see the baseline of thick real curve with $p_{1}=1$ in Fig. 9b), meanwhile, there are also spare beds in FSH from February 17 (see the thin dash curve with $p_{1}=0.95$ in Fig. 9c). In addition, one can also observe in Fig. 9 that, if the voluntary home quarantine was not effectively implemented, then the spread of COVID-19 in Wuhan would be out of control with the actual available hospital beds in DH and FSH (see the dot curves with $p_{1}=1.1$ and thick dash curves with $p_{1}=1.05$ in Fig. 9a-c).

Figure 10 performs sensitivity analysis of home quarantine under different hypothetical scenarios of hospital bed capacity in Wuhan. In Fig. 10a, the hospital bed capacities in both DH and FSH are assumed to be one and a half times of the actual available beds (i.e., $1.5 B_{D H}(t)$ and $1.5 B_{M H}(t)$ ), then the spread of COVID-19 in Wuhan would be still finally inhibited even if the numbers of close contact are increased by $10 \%$ (see the dot curve with $p_{0}=1.5$ and $p_{1}=1.1$ ). However, when the corresponding parameters are increased by $20 \%$ or $30 \%$, the transmission would be out of control (see the dash-dot curve with $p_{0}=1.5, p_{1}=1.2$ and dash curve with $\left.p_{0}=1.5, p_{1}=1.3\right)$. In Fig. $10 \mathrm{~b}$, one can find that, if the available beds double the actual number (i.e., $2 B_{D H}(t)$ and $2 B_{M H}(t)$ ), then the spread of COVID-19 in Wuhan would be controlled finally when the numbers of close contact are increased by $20 \%$ (see the dash-dot curve with $p_{0}=2$ and $p_{1}=1.2$ ). If the parameters $\sigma^{I}, \sigma^{Q}$, and $\sigma^{A}$ are increased by $30 \%$, then even doubling the actual number of available hospital beds still cannot inhibit the spread of COVID-19 in Wuhan (see the dash curve with $p_{0}=2$ and $p_{1}=1.3$ in Fig. 10b).

In Fig. 11, we carry out numerical simulations for the dynamics of cumulative confirmed cases under different hypothetical scenarios of home quarantine efficiency and hospital bed capacity in Wuhan. Figure 11a reveals that, compared with FSH, DH performs more effectively on the containment of COVID-19 in Wuhan. As of March 10, all available hospital beds in FSH averted about 80,000 new confirmed cases (see the heavy dash curve with $p_{0}^{D}=1, p_{0}^{M}=0, p_{1}=1$, and the baseline in Fig. 11a), while one half of available beds in DH averted more than 110,000 new confirmed 


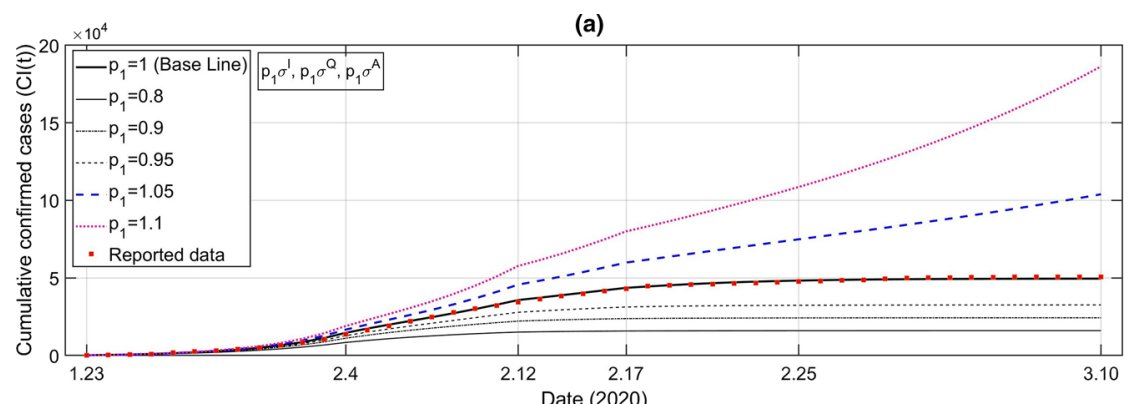

(b)

Date (2020)

(c)
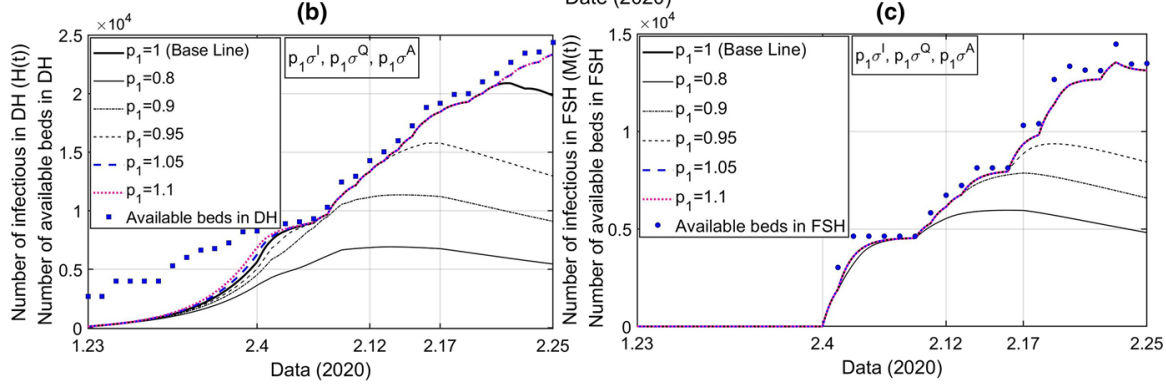

Fig. 9 Sensitivity analysis of home quarantine on the containment of COVID-19 in Wuhan in terms of $\sigma^{I}$, $\sigma^{Q}$, and $\sigma^{A}$. The values of $\sigma^{I}, \sigma^{Q}$, and $\sigma^{A}$ are changed by adjusting parameter $p_{1}$, while other parameters are the same as those in Fig. 5
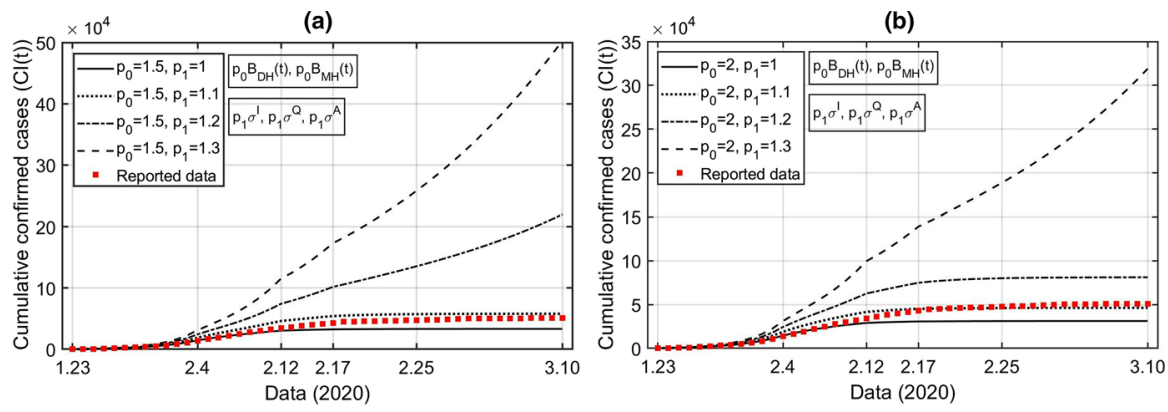

Fig. 10 Sensitivity analysis of home quarantine under different hypothetical scenarios of hospital bed capacity in Wuhan in terms of $\sigma^{I}, \sigma^{Q}$, and $\sigma^{A}$. The values of $B_{D H}(t), B_{M H}(t)$, and $\sigma^{I}, \sigma^{Q}, \sigma^{A}$ are changed by adjusting parameters $p_{0}$ and $p_{1}$, respectively, while other parameters are the same as those in Fig. 5

cases (see the dash curve with $p_{0}^{D}=0.5, p_{0}^{M}=1, p_{1}=1$, and the baseline in Fig. 11a). If there is no FSH, with the increasing of $80 \%$ beds in $\mathrm{DH}$ (i.e., $1.8 B_{D H}(t)$ ), the spread of COVID-19 in Wuhan still could be inhibited and the cumulative confirmed cases are even reduced to about 40,000 (see the heavy dot curve with $p_{0}^{D}=1.8$, $p_{0}^{M}=0$ and $p_{1}=1$ in Fig. 11a). However, with one half of available beds in DH (i.e., $0.5 B_{D H}(t)$ ), one needs three times the number of actual available beds in FSH (i.e., $\left.3 B_{M H}(t)\right)$ to reduce the cumulative confirmed cases to an approximate number (see the red dot curve with $p_{0}^{D}=0.5, p_{0}^{M}=3$ and $p_{1}=1$ in Fig. 11a). Simulations in Fig. $11 \mathrm{~b}$ also indicate that, in addition to $\mathrm{DH}$ and FSH, home quarantine of close 

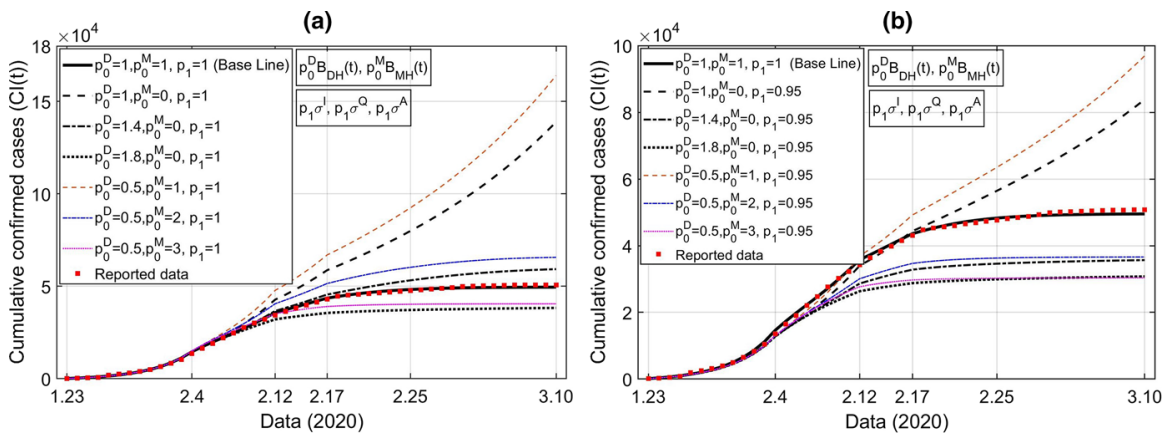

Fig. 11 Comparisons of cumulative confirmed cases under different hypothetical scenarios of home quarantine efficiency and hospital bed capacity in Wuhan. The values of $B_{D H}(t), B_{M H}(t)$, and $\sigma^{I}, \sigma^{Q}, \sigma^{A}$ are changed by varying parameters $p_{0}^{D}, p_{0}^{M}$ and $p_{1}$, respectively, while others are the same as those in Fig. 5

contact is another effective control measure. If FSH was not adopted in Wuhan, then decreasing $5 \%$ of the number of close contact (i.e., $\left.p_{1}=0.95\right)$ together with increase of $40 \%$ beds in $\mathrm{DH}$ (i.e., $\left.1.4 B_{D H}(t)\right)$ could effectively control the transmission of COVID-19 in Wuhan and reduces the cumulative confirmed case to less than 40,000 (see the heavy dash-dot curve with $p_{0}^{D}=1.4, p_{0}^{M}=0$ and $p_{1}=0.95$ in Fig. 11b).

\subsection{Effect of Tracing Efficiency in Early Stage of the Outbreak}

To investigate the impact of the contact tracing efficiency during Stage I, in Fig. 12, sensitivity analysis is carried out using the parameterized model with different values of $\tau^{I}, \tau^{Q}$, and $\tau^{A}$ in the corresponding period (i.e., from January 23 to February 4). One can find in Fig. 12 that, in Stage I, all simulated curves have similar values and increasing trends, yet the number of cumulative confirmed cases shows great differences over time in the following stages. If the tracing rate in Stage I was increased by $20 \%$ (i.e., $p_{2}=1.2$ ), then the cumulative confirmed cases would be averted more than 20,000 cases (see the dash curve with $p_{2}=1.2$ in Fig. 12). On the other hand, if the tracing rate in Stage I is decreased by $20 \%$ (i.e., $p_{2}=0.8$ ), the total number of cumulative confirmed cases in Wuhan would be far more than the current infection size (see the thick dot curve with $p_{2}=0.8$ and the baseline of thick real curve with $p_{2}=1$ in Fig. 12). In comparison, sensitivity analysis of the detection rate in Stage II (i.e., from February 5 to 12) is shown in Fig. 13, which demonstrates that high tracing rate in Stage II had much less effect on the control of COVID-19 than that in Stage I.

\section{Discussion and Conclusion}

Now, the COVID-19 pandemic has become a major public health emergency. The disease has spread faster and wider around the world than any others in the past decades, and has also proven to be the most difficult to control. The Wuhan lockdown and its success in containing of the epidemic provides a valuable experience for other regions of the world. 


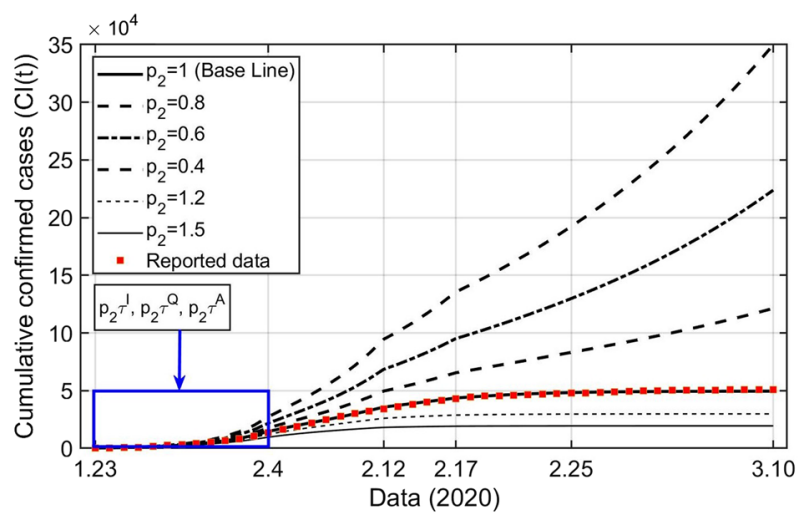

Fig. 12 Sensitivity analysis of tracing efficiency in Stage I (i.e., from January 23 to February 4) on the containment of COVID-19 in Wuhan. The values of $\tau^{I}, \tau$, , and $\tau^{A}$ in Stage $I$ are changed by adjusting parameter $p_{2}$, while other parameters are the same as those in Fig. 5

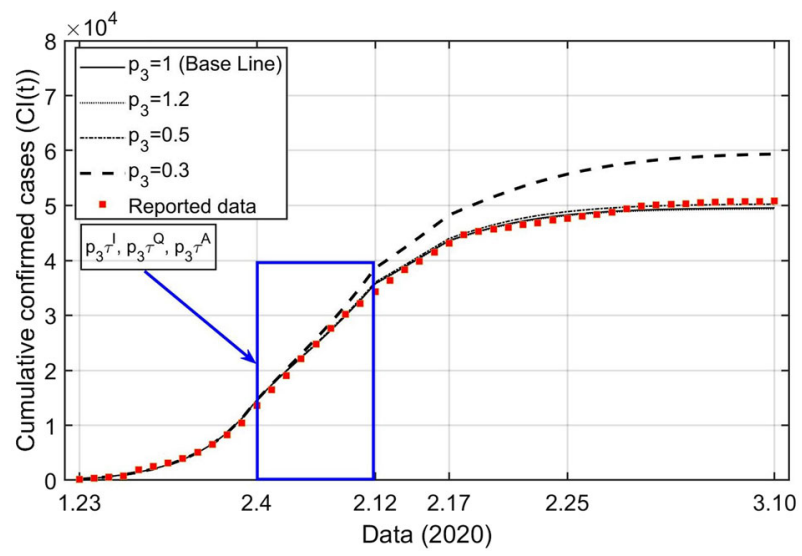

Fig. 13 Sensitivity analysis of tracing efficiency in Stage II (i.e., from February 5 to February 12) on the containment of COVID-19 in Wuhan. The values of $\tau^{I}, \tau^{Q}$, and $\tau^{A}$ in Stage II are changed by adjusting parameter $p_{3}$, while other parameters are the same as those in Fig. 5

In this study, we established a dynamical model by considering four different stages of transmission. Several time-dependent parameters and four stages of the outbreak were used to reflect the changes of medical resources and the timely adjustments of control strategies on the transmission of COVID-19 in Wuhan. Then, we proposed a dynamics-based data inversion method to calibrate the under-reported infections accumulated from clinically diagnosed cases before February 12. Compared with Lin et al. (2020), our method follows the evolution pattern generated by a parameterized dynamic model and avoids enlargement of data collection biases caused by various reasons. Based on the reported data of cumulative confirmed cases, death cases, and recovered cases in Wuhan from January 23 to February 25, the time-varying transmission model is parameterized by employing nonlinear least squares method. It is worth mentioning that, the model simulation shows reasonably good match with the 
multi-dimensional real data and makes a good prediction of the cumulative confirmed cases from February 26 to March 10 (see Fig. 5), in particular, the simulation-based recurrence time point of spare beds in DH (see Fig. 6a) is well consistent with the actual situation (Health Commission of Hubei Province 2020d).

It is well known that the prevention and control of infectious disease follow the classic CCP principle, i.e., control the sources infection, cut the route of transmission, and protect the vulnerable group. When one revisits the transmission process of COVID19 in Wuhan, the JPCM plays a decisive role on the containment of COVID-19, which contains a series of integrated and coordinated actions such as contact tracing, home quarantine, and building of DH and FSH. Our four-stage nonautonomous model reveals that the spread of COVID-19 cannot be inhibited in Wuhan if any of these measures is not effectively implemented (see Figs. 7, 8, 9, 10, 11, 12, 13). Furthermore, numerical analysis provides a scientific approach to understand how these actions work comprehensively on controlling COVID-19 in Wuhan.

FSH is one of the most important and impressive strategies in the success of tackling the COVID-19 transmission in Wuhan. In early February 2020, with the shortage of beds available for the treatment of the infectious, there were thousands of mild infected patients sent home for self-quarantine and observation. However, home quarantine could put patient's family members at high risk. Although the hospital beds in DH were continuously increasing, the epidemic was getting worse till the FSH was adopted and mainly served to isolate and care for patients with mild symptoms. When most of the infectious with mild symptoms and their close contacts were moved to FSH, the household transmission was greatly reduced. The number of reported confirmed cases steadily decreased from February 18, 12 days after the first FSH started admitting patients. The FSH played an important role as expected (Routine press conference on the prevention and control of the COVID-19 epidemic 2020) and many countries had already adopted similar approaches for the containment of COVID-19 (Li et al. 2020c).

Nevertheless, our analysis suggests another fact that FSH is just very helpful replenishment when the bed capacity of DH cannot be increased to necessary level in the short term. Comprised with FSH, the DH can inhibit the transmission of COVID-19 more effectively. Figures 9, 10, and 11 reveal that the effective home quarantine is also a decisive factor for success in containment of COVID-19 in Wuhan. If people did not stay at home and most traditional family gatherings for the new year festival were not stopped, a small increase in the number of close contact would lead to increasing trend of COVID-19 in Wuhan. Low tracing efficiency of infectious in the early stage of outbreak would cause a large number of new infections, and then the cumulative number of confirmed cases in the following stages would be beyond imagination (see Fig. 12). That is to say, timely and quickly tracing of infectious at the beginning of outbreak is crucial for controlling of COVID-19, although it may need a huge economic cost. Furthermore, the outbreaks in other regions of China, such as the outbreak occurred in Beijing on June 11, 2020 (National Health Commission of the People's Republic of China 2020a), and the outbreak lighted in Hebei Province on January 3, 2021 (Health Commission of Hebei Province 2021), were also effectively constrained by the insights of JPCM. The World Health Organization stated that China has paid enormous efforts in responding to the COVID-19 epidemic and has effectively pre- 
vented the further spread of the epidemic. In particular, the prevention and control measures implemented in Wuhan and other infected cities were timely and effective. The World Health Organization also calls on other countries and regions in the world to learn from China (World Health Organization 2021). In summary, the combining multiple measures must be simultaneously implemented as quickly and as early as possible when we struggle with COVID-19 or other pandemic in future.

Acknowledgements The research was supported by National Natural Science Foundation of P. R. China (Nos. 12071068, 12126206, 12026202, 1210010988), the Natural Science Foundation of Jilin Province (No. 20210101482JC), and partially by the 2021 Provincial Natural Science Foundation Joint Guidance Project of Heilongjiang Province (LH2021A003).

\section{Declaration}

Conflict of interest The authors declare that they have no conflict of interest.

\section{References}

Alaran AJ, Adebisi YA, Badmos A, Khalid-Salako F, Gaya SK, Ilesanmi EB, Olaoye DQ, Bamisaiye A, Lucero-Prisno DE (2021) Uneven power dynamics must be levelled in COVID-19 vaccine access and distribution. Public Health Pract 2:100096

Chen C, Liao Z, Chmura SJ, Weichselbaum RR (2020a) COVID-19 infection prevention and control practices in Wuhan radiotherapy. Adv Radiat Oncol 5(4):637-639

Chen SM, Zhang ZJ, Yang JT, Wang J, Zhai XH, Bärnighausen T, Wang C (2020b) Fangcang shelter hospitals: a novel concept for responding to public health emergencies. The Lancet 395(10232):13051314

Chen N, Zhou M, Dong X, Qu J, Gong F, Han Y, Qiu Y, Wang J, Liu Y, Wei Y, Xia J, Yu T, Zhang X, Zhang L (2020c) Epidemiological and clinical characteristics of 99 cases of 2019 novel coronavirus pneumonia in Wuhan, China: a descriptive study. Lancet 395(10223):507-513

Eaton L (2021) Covid-19: WHO warns against "vaccine nationalism" or face further virus mutations. BMJ 372:n292

Fang HM, Wang L, Yang Y (2020) Human mobility restrictions and the spread of the novel coronavirus (2019-nCoV) in China. J Public Econ 191:104272

Hao XJ, Cheng SS, Wu DG, Wu TC, Lin XH, Wang CL (2020) Reconstruction of the full transmission dynamics of COVID-19 in Wuhan. Nature 584:420-4

Health Commission of Hubei Province (2020a) Epidemic cases. http://wjw.hubei.gov.cn/fbjd/tzgg/202001/ t20200123_2014525.shtml. Accessed 15 May 2021

Health Commission of Hubei Province (2020b) Prevention and control of COVID-19, Huoshenshan Hospital. http://wjw.hubei.gov.cn/bmdt/ztzl/fkxxgzbdgrfyyq/fkdt/202003/t20200317_2183343. shtml. Accessed 15 May 2021

Health Commission of Hubei Province (2020c) Prevention and control of COVID-19, Leishenshan Hospital. http://wjw.hubei.gov.cn/bmdt/ztzl/fkxxgzbdgrfyyq/fkdt/202004/t20200417_2232817. shtml. Accessed 15 May 2021

Health Commission of Hubei Province (2020d) Prevention and Control of Novel Coronavirus Pneumonia. http://wjw.hubei.gov.cn/bmdt/ztzl/fkxxgzbdgrfyyq/fkdt/202002/t20200215_2028454.shtml. Accessed 07 May 2021

Health Commission of Hebei Province (2021) Instructions for the prevention and control of the COVID-19 epidemic in Hebei Province. http://wsjkw.hebei.gov.cn/html/tzgg/20210121/375771.html. Accessed 26 May 2021

Hubei Daily (2020) Centralized isolation of four categories of personnel. Wuhan Municipal Health Commission. http://wjw.wuhan.gov.cn/ztzl_28/fk/fkdt/202004/t20200430_1197006.shtml. Accessed 05 Aug 2021 
Huo X, Chen J, Ruan S (2020) Estimating asymptomatic, undetected and total cases for the COVID-19 outbreak in Wuhan: a mathematical modeling study. BMC Infect Dis 21:476

Kraemer MUG, Yang CH, Gutierrez B, Wu CH, Klein B, Pigott DM, Plessis L, Faria NR, Li R, Hanage WP, Brownstein JS, Layan M, Vespignani A, Tian H, Dye C, Pybus OG, Scarpino SV (2020) The effect of human mobility and control measures on the COVID-19 epidemic in China. Science 368(6490):493497

Kucharski AJ, Klepac P, Conlan AJK, Kissler SM, Tang ML, Fry H, Gog JR, Edmunds WJ (2020) Effectiveness of isolation, testing, contact tracing, and physical distancing on reducing transmission of SARS-CoV-2 in different settings: a mathematical modelling study. Lancet Infect Dis 20(10):11511160

Leung K, Wu JT, Liu D, Leung GM (2020) First-wave COVID-19 transmissibility and severity in China outside Hubei after control measures, and second-wave scenario planning: a modelling impact assessment. The Lancet 395(10233):1382-1393

Li Q, Guan X, Wu P, Wang XY, Zhou L, Tong YQ, Ren RQ, Leung KSM, Lau EHY, Wong JY, Xing XS, Xiang NJ, Wu Y, Li C, Chen Q, Dan Li, Liu T, Zhao J, Li M, Tu WX, Chen CD, Jin LM, Yang R, Wang Q, Zhou SH, Wang R, Liu H, Luo YB, Liu Y, Shao G, Li H, Tao ZF, Yang Y, Deng ZQ, Li BX, Ma ZT, Zhang YP, Shi GQ, Lam TTY, Wu JTK, Gao GF, Cowling BJ, Yang B, Leung GM, Feng ZJ (2020a) Early transmission dynamics in Wuhan, China, of novel coronavirus infected pneumonia. N Engl J Med 382(13):1199-1207

Li Q, Guan X, Wu P, Wang X, Zhou L, Tong Y, Ren R, Leung KSM, Lau EHY, Wong JY, Xing X, Xiang N, Wu Y, Li C, Chen Q, Li D, Liu T, Zhao J, Liu M, Tu W, Chen C, Jin L, Yang R, Wang Q, Zhao S, Wang R, Liu H, Lou Y, Liu Y, Shao G, Li H, Tao Z, Yang Y, Deng Z, Liu B, Ma Z, Zhang Y, Shi G, Lam TTY, Wu JT, Gao GF, Cowling BJ, Yang B, Leung GM, Feng Z (2020b) Early transmission dynamics in Wuhan, China, of novel coronavirus infected pneumonia. N Engl J Med 382:1199-1207

Li J, Yuan P, Heffernan J, Zheng T, Ogden N, Sander B, Li J, Li Q, BšŠlair J, Kong JD, Aruffo E, Tan Y, Jin Z, Yu Y, Fan M, Cui J, Teng Z, Zhu H (2020c) Fangcang shelter hospitals during the COVID-19 epidemic, Wuhan. China. Bull World Health Organ 98(12):830-841D

Li XH, Zhao XM, Sun YH (2020d) The lockdown of Hubei province causing different transmission dynamics of the novel coronavirus (2019-ncov) in Wuhan and Beijing. medRxiv

Lin QY, Zhao S, Gao DZ, Lou YJ, Yang S, Musa SS, Wang MH, Cai YL, Wang WM, Yang L, He DH (2020) A conceptual model for the coronavirus disease 2019 (COVID-19) outbreak in Wuhan, China with individual reaction and governmental action. Int J Infect Dis 93:211-216

Liu Z, Magal P, Seydi O, Webb G (2020a) Understanding unreported cases in the COVID-19 epidemic outbreak in Wuhan, China, and the importance of major public health interventions. Biology 9(3):50

Liu M, Ning J, Du YR, Cao J, Zhang D, Wang JG, Chen MJ (2020b) Modeling the evolution trajectory of COVID-19 in Wuhan, China: experience and suggestions. Public Health 183:76-80

Matteo C, Jessica TD (2020) The effect of travel restrictions on the spread of the 2019 novel coronavirus (2019-ncov) outbreak. Science 368(6489):395-400

Ming W, Huang J, Zhang C J P (2020) Breaking down of healthcare system: mathematical modelling for controlling the novel coronavirus (2019-ncov) outbreak in Wuhan, China. bioRxiv

National Health Commission of the People's Republic of China (2020a) Instructions on the prevention and control of the COVID-19 epidemic in Beijing. http://www.nhc.gov.cn/wjw/xwdt202006/ 12602535945640248effa18b056d4256.shtml. Accessed 26 May 2021

National Health Commission of the People's Republic of China (2020b) The latest situation of new coronavirus pneumonia. http://www.nhc.gov.cn/xcs/yqtb/202002/26fb16805f024382bff1de80c918368f. shtml. Accessed 07 May 2021

Nørgaard SK, Vestergaard LS, Nielsen J, Richter L, Schmid D, Bustos N, Braye T, Athanasiadou M, Lytras T, Denissov G, Veideman T, Luomala O, Möttönen T, Fouillet A, Caseri-Schönemann C, an der Heiden M, Uphoff H, Gkolfinopoulou K, Bobvos J, Paldy A, Rotem N, Kornilenko I, Domegan L, O’Donnell J, Donato F D, Scortichini M, Hoffmann P, Velez T, England K, Calleja N, van Asten L, Stoeldraijer L, White R A, Paulsen T H, da Silva S P, Rodrigues A P, Klepac P, Zaletel M, Fafangel M, Larrauri A, León I, Farah A, Galanis I, Junker C, Perisa D, Sinnathamby M, Andrews N, O’Doherty M G, Irwin D, Kennedy S, Mcmenamin J, Adlhoch C, Bundle N, Penttinen P, Pukkila J, Pebody R, Krause T G, Mølbak K, (2021) Real-time monitoring shows substantial excess all-cause mortality during second wave of COVID-19 in Europe, October to December 2020. Eurosurveillance 26(2):2002023 
Office of Health Emergency (2020) The latest situation of new coronavirus pneumonia. National Health Commission of the People's Republic of China. http://www.nhc.gov.cn/xcs/yqtb/202003/ e644c2fc18b4448db7ed4b30f68b91a6.shtml. Accessed 05 Aug 2021

Pan A, Liu L, Wang C, Guo H, Hao X, Wang Q, Huang J, He N, Yu H, Lin X, Wei S, Wu T (2020) Association of public health interventions with the epidemiology of the COVID-19 outbreak in Wuhan, China. JAMA 323(19):1915-1923

Pang L, Liu S, Zhang X, Tian T, Zhao Z (2020) Transmission dynamics and control strategies of COVID-19 in Wuhan, China. J Biol Syst 28(462):1-18

People's Network (2020) Where are the more than 5 million people who left Wuhan? These three places are the most. http://society.people.com.cn/GB/n1/2020/0128/c1008-31563429.html. Accessed 17 Feb 2020

Prevention and Control Dynamics, Xinhuanet (2020) Central to Hubei steering group: all should be treated without delay. Wuhan Municipal Health Commission. http://wjw.wuhan.gov.cn/ztzl_28/fk/ fkdt/202004/t20200430_1197064.shtml. Accessed 05 Aug 2021. Cartwright J (2007) Big stars have weather too. IOP Publishing PhysicsWeb. http://physicsweb.org/articles/news/11/6/16/1. Accessed 26 June 2007

Robinson J, Banerjee I, Leclézio A, Sathian B (2021) COVID-19 and mutations a threat level assessment. Nepal J Epidemiol 11(1):983-987

Rong XM, Yang L, Chu HD, Fan M (2020) Effect of delay in diagnosis on transmission of COVID-19. Math Biosci Eng 17(3):2725-2740

Routine press conference on the prevention and control of the COVID-19 epidemic (2020) Wuhan Municipal Health Commission. http://wjw.wuhan.gov.cn/ztzl_28/fk/fkdt/202004/t20200430_1198869.shtml. Accessed 15 May 2021

Sun GQ, Wang SF, Li MT, Li L, Zhang J, Zhang W, Jin Z, Feng GL (2020) Transmission dynamics of COVID-19 in Wuhan, China: effects of lockdown and medical resources. Nonlinear Dyn 101:19811993

Sun S, Lin D, Operario D (2021) Interest in COVID-19 vaccine trials participation among young adults in China: Willingness, reasons for hesitancy, and demographic and psychosocial determinants. Prevent Med Rep 1:101350

Tang B, Wang X, Li Q, Bragazzi NL, Tang S, Xiao Y, Wu J (2020) Estimation of the transmission risk of the 2019-nCoV and its implication for public health interventions. J Clin Med 9(2):462

The Central People's Government of China (2020) http://politics.cntv.cn/special/gwyvideo/2019/202002/ 2020021701/index.shtml. Accessed 18 May 2021

The State Council Information Office of the People's Republic of China (2020) Fighting COVID-19: China in Action. http://www.scio.gov.cn/zfbps/32832/Document/1681809/1681809.htm. Accessed 07 May 2021

Wang L, Zhou Y, He J, Zhu B, Song PXK (2021) An epidemiological forecast model and software assessing interventions on COVID-19 epidemic in China. J Data Sci 18(3):409-432

World Health Organization (2021) Coronavirus (COVID-19) dashboard. https://covid19.who.int. Accessed 26 May 2021

Xin X, Li SF, Cheng L, Liu CY, Xin YJ, Huang HL, Beejadhursing R, Wang SS, Feng L (2021) Government intervention measures effectively control COVID-19 epidemic in Wuhan, China. Curr Med Sci 41:7783

Yan J, Zhao D (2020) Administrative mechanism of Joint Participation and Cooperation in the early stages of the COVID-19 outbreak in Wuhan. Risk Manag Healthc Policy 13:723-731

Yang Y, Lu Q B, Liu M J, Wang Y X, Zhang A R, Jalali N, Dean N E, Longini I, Halloran M E, Xu B, Zhang X A, Wang L P, Liu W, Fang L Q (2020) Epidemiological and clinical features of the 2019 novel coronavirus outbreak in China. medRxiv

Zhao S, Musa SS, Lin Q, Ran J, Yang G, Wang W, Lou Y, Yang L, Gao D, He D, Wang MH (2020) Estimating the unreported number of novel coronavirus (2019-nCoV) cases in China in the first half of January 2020, a data-driven modelling analysis of the early outbreak. J Clin Med 9(2):388

Publisher's Note Springer Nature remains neutral with regard to jurisdictional claims in published maps and institutional affiliations. 


\section{Authors and Affiliations}

Linhua Zhou ${ }^{3} \cdot$ Xinmiao Rong ${ }^{1,4} \cdot$ Meng Fan $^{1}$ (D) Liu Yang ${ }^{1} \cdot$ Huidi Chu ${ }^{1}$. Ling $\mathrm{Xue}^{4}$. Guorong $\mathrm{Hu}^{1}$. Siyu Liu ${ }^{5}$. Zhijun Zeng ${ }^{1}$ - Ming Chen ${ }^{7}$. Wei Sun ${ }^{3}$.

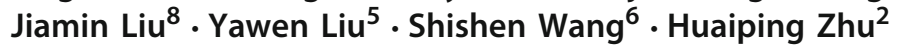

$\bowtie \quad$ Meng Fan

mfan@nenu.edu.cn

$凶$ Huaiping Zhu

Huaiping@yorku.ca

1 School of Mathematics and Statistics, Northeast Normal University, Changchun, China

2 Center for Disease Modelling, York University, Toronto, Canada

3 School of Science, Changchun University of Science and Technology, Changchun, China

4 College of Mathematical Sciences, Harbin Engineering University, Harbin, China

5 Jilin University, Changchun, China

6 Changchun Center for Disease Control and Prevention, Changchun, China

7 School of Science, Dalian Maritime University, Dalian, China

8 School of Mathematics, Harbin Institute of Technology, Harbin, China 